\title{
Artificial Intelligence in Trading the Financial Markets
}

Submitted 18/11/21, 1st revision 11/12/21, 2nd revision 12/01/22, accepted 10/02/22

\begin{abstract}
Gil Cohen ${ }^{1}$
Abstract:

Purpose: The purpose of this study is to review the methods that are used to construct Artificial Intelligence (AI) algorithmic trading systems.

Design/Methodology/Approach: A review approach of the existing knowledge was used.

Findings: We find that there are various methodologies that are used by researchers and practitioners when they contract algorithmic trading systems. Some of the systems combine data from the financial markets alone and some methods combine financial data with social media data. The ability of computerized algorithms to integrate a large set of data and react almost immediately according to it, does not come without risks of accelerating downtrends in times of panic in the financial market and therefore those systems must be institutionally monitored by the regulating authorities.

Practice Implication: This study enables readers to understand the major methodologies that are used to predict trends of financial assets prices. The research identifies and explains the complexity of methods that helps traders to improve their trading results.

Originality Value: No past study has summarized the major methodologies that are used to construct and optimize trading results.
\end{abstract}

Keywords: Algorithmic, trading, technical analysis, artificial intelligence.

JEL Codes: G24, G41, G17.

Paper type: A review paper.

${ }^{1}$ Prof., Western Galilee College, Acre, Israel, gilc@wgalil.ac.il; 


\section{Introduction}

Machine Learning (ML) and Big Data has become a popular research tool for locating and trading securities. Those algorithms usually but not exclusively combine financial data that were extracted from the financial markets with social media data. Today about $80 \%$ of trades are made by high frequency machines that unfairly compete with human traders in terms of decision making and order generation speed.

Another important aspect of this revolution is that trained machines do not suffer from human bias and emotions that control a big portion of their investment decision making. The modern algorithmic trading systems make use of many years of experiences of traders and market researchers combined with the newly advances in ML and autonomous pattern recognition. Moreover, some systems use data from social media combined with market data to predict financial assets trends. This paper summarizes the major methodologies that exist in the attempt to forecast financial assets price trends.

\section{Social Media and Trading}

Social media can supply important data to investors' mood regarding the different financial assets that are traded on the markets. The progress and popularity of social media enable investors from every age group and trading experience to find their way to communicate with each other and exchange trading ideas in real time. Liu (2020) investigates whether content from social media has differential impacts on stock performance. They collected a large dataset of 84 million tweets and 8 years of stock data for 407 companies from the S\&P 500 index and found that while consumers' positive sentiment does not play a significant role in stock performance, consumers' negative sentiment significantly impacts stock prices.

Sohangir et al. (2018) applied several neural network models such as long short-term memory and convolutional neural networks, to stock market opinions posted in stock twits. Their results show that the Deep Learning model can be used effectively for financial sentiment analysis and a convolutional neural network is the best model to predict the sentiment of authors in stock twit's dataset. Since financial asset price movements are very complex, non-stationary and nonlinear, they are affected by many factors, making it difficult to predict it with a simple model.

\section{Support Vector Machines}

Support Vector Machines (SVM) are widely used as data mining methods in the field of machine learning. SVM, developed by Vapnik (1995) is an artificial intelligence learning method. It is a machine learning technique based on statistical learning theory and structural risk minimization. The purpose is to identify the optimal separating hyperplane to divide two or more classes of data with the 
learning mechanism by training the input data. SVM is a type of supervised learning to predict and classify items and it is well known as a useful machine learning algorithm for classification (Jan, 2018). The important advantage of SVM is that it allows error within the regression of training data so that the error in the test data is reduced significantly.

In addition to SVMs, Long-Short Term Memory (LSTM) has also seen increased usage recently. The major advantage of LSTMs is that it can learn selectively and can remember or forget the required historical data. Xiao et al. (2020) analyzes the shortcomings of stock market forecasting methods using standard SVM firstly, at the same time, the cumulative auto-regressive moving average is proposed, which combines the least squares support vector machine synthesis model to make basic predictions for the stock market. They concluded that the combined model based is more suitable for stock price forecasting than the single forecasting model, and the actual performance is better.

Nelson et al. (2017) studied the usage of LSTM networks to predict future trends of stock prices based on the price history, alongside with technical analysis indicators. For that goal, a prediction model was built, and a series of experiments were executed and their results analyzed against several metrics to assess if this type of algorithm presents improvements when compared to other ML methods and investment strategies. The results that were obtained are promising, getting up to an average of $55.9 \%$ of accuracy when predicting if the price of a particular stock is going to go up or not soon. Borovkova and Tsiamas (2019) ensembled LTSM model for intraday stock predictions, using a large variety of technical analysis indicators as network inputs. They evaluate the predictive power of our model on several US large-cap stocks and benchmark it against lasso and ridge logistic classifiers.

\section{Artificial Neural Networks}

Researchers have also suggested that Artificial Neural Networks (ANN) provide an opportunity to achieve profits exceeding the market average by using technical indicators as predictors in stock markets (Fischer, 2018; Lee 2013). Shin et al. (2017) proposed a stock price prediction model based on deep learning techniques using open-high-low-close price and volume and derived technical indicators in the Korean stock market. The ANN, as a deep learning technique, is a model that imitates the visual processing of living organisms that recognize patterns or images.

The network learns filters that activate when they see some type of visual feature, such as an edge of some orientation of the first layer or eventually the entire honeycomb or wheel-like patterns within the higher layers of the network. An entire set of filters is generated in each convolutional layer, and each one produces a separate 2-dimensional feature map that sends real time information to the ANN system and makes automated financial decisions according to a predetermined algorithm. 


\section{Technical Analysis and Pattern Recognition}

Technical analysis helps investors to better time their entry and exit from financial asset positions. This methodology relies solely on past information of financial assets price and volumes to predict a financial asset future price trend. Modern research has established that combined with other sentiment measures such as social media, it can outperform the standard buy and hold strategy.

Moreover, it has been documented that novice and professional investors use technical analysis in their investing strategy. An experienced investor should combine fundamental analysis and technical analysis for better trading results. Programmers use technical analysis to create algorithmic trading systems that learn and adapt to the changing trading environments and perform trading accordingly without human involvement. There are hundreds of technical tools offered by known trading platforms. investors must use specific tools that fits his trading style and risk adoption. Moreover, different financial assets such as stocks, ETFs, Cryptocurrency, Futures, and commodities demand different sets of tools. Furthermore, investors should use these tools according to the time frame they use for trading.

\subsection{Pattern Recognition}

An important aspect of technical analysis is Pattern Recognition (PR) which is a well known AI tool in which disturbances are located within an observed phenomenon. In Finance PR is used in two main aspects. First, to locate a trend line breaks of an uptrend or downtrend and candlesticks pattern recognitions that are widely used to locate relatively quick trend shifts. Figure 1 shows the fluctuation of a stock price over a period. Each candlestick represents one trading day.

\section{Figure 1. Support and Resistance Lines}

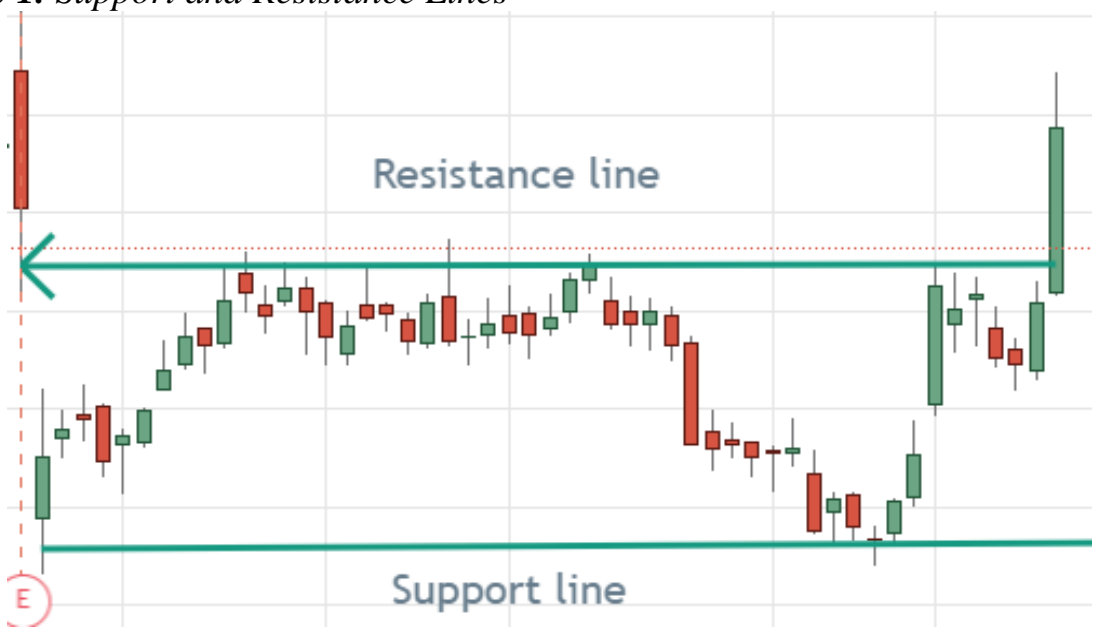

Source: Own study. 
Figure 1 shows that once the stock price dived down it stopped at a specific low price twice in the examined period. That means that buyers assume that at the support level it is a good price to buy and by doing so they are sending the stock to an uptrend. On the other hand, when the price reaches a specific high price, the sellers become dominant, sending the price back down. This behavior of buyers and sellers is repetitive until a breakout occurs that starts a new up or down trend. This example explains the nature of technical trading in general, it is based on trader's psychology and behavior. Support and resistance lines do not have to be horizontal; they can also be diagonals creating a channel that characterizes the financial asset price movement as can be viewed in Figure 2.

\section{Figure 2. Diagonal Support and Resistance Channel}

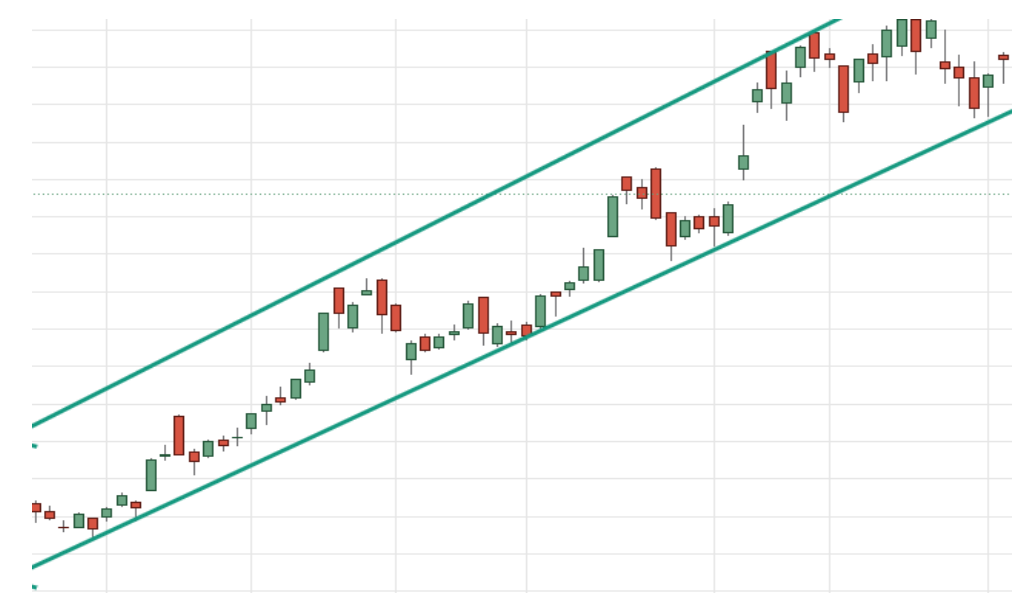

Source: Own study.

The channel shape demonstrated in Figure 2 shows an uptrend of the financial asset. An experienced trader would buy the asset near the support level and sell near the resistance level.

\subsection{Darvas Boxes}

Darvas box pattern was invented by Nicolas Darvas in the 1950s while traveling the world as a professional ballroom dancer. His trading technique involves drawing a box around the recent highs and lows of the financial asset to establish entry point and placement of the stop-loss order. The theory uses market momentum theory along with technical analysis to determine when to enter and exit the market. Darvas believed his method worked best when applied to industries with the greatest potential to excite investors and consumers with revolutionary products. The fact that Bitcoin is relatively new and revolutionary in terms of a digital currency without a central bank or a single other administrator or intermediaries, has fascinated investors around the globe. 
Those characteristics make it a perfect candidate for price trends forecasting using Darvas Boxes technique. Figure 3 shows entries to long and short positions using Darvas Boxes technique.

Figure 3. Darvas Box Trading Strategy

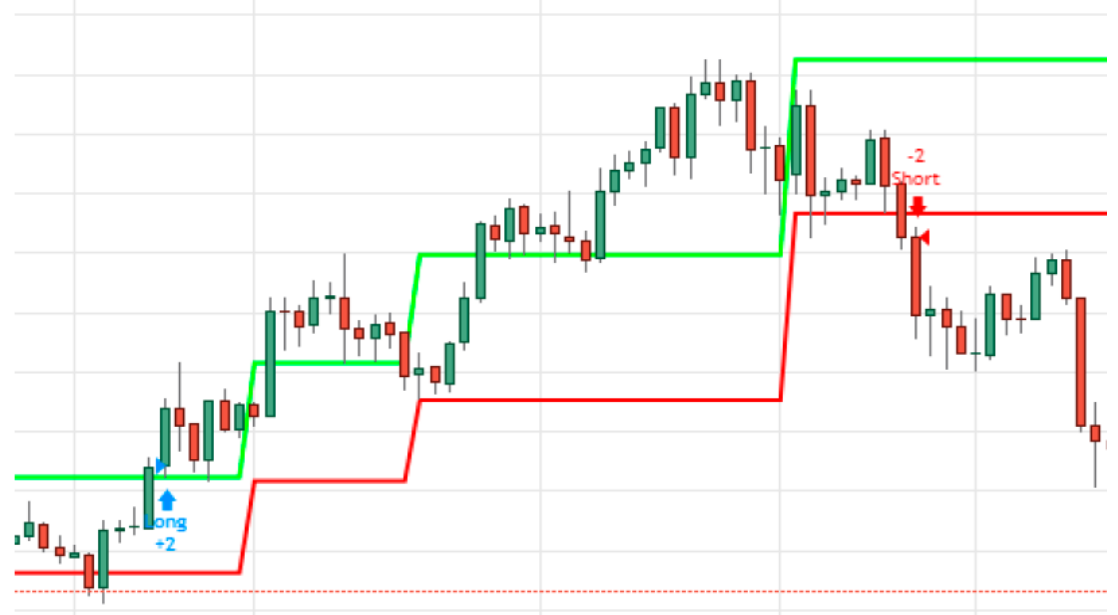

Source: Own study.

Figure 3 shows an entry to a long position when the price breaks up the box formation and an entry to a short position when price breaks down the box formation.

\subsection{Linear Regression}

Linear regression is a supervised learning technique typically used in predicting, forecasting, and finding relationships between quantitative data. It is one of the earliest learning techniques, which is still widely used. A Linear Regression line is a straight line that best fits the prices between a starting price point and an ending price point. A "best fit" means that a line is constructed where there is the least amount of space between the price points and the actual Linear Regression line.

When prices are below the Linear Regression Line, this could be viewed by some traders as a good time to buy, and when prices are above the Linear Regression line, a trader might sell. Figure 4 demonstrates the trading technique using Linear Regression. 
Figure 4. Linear Regression Trading Strategy

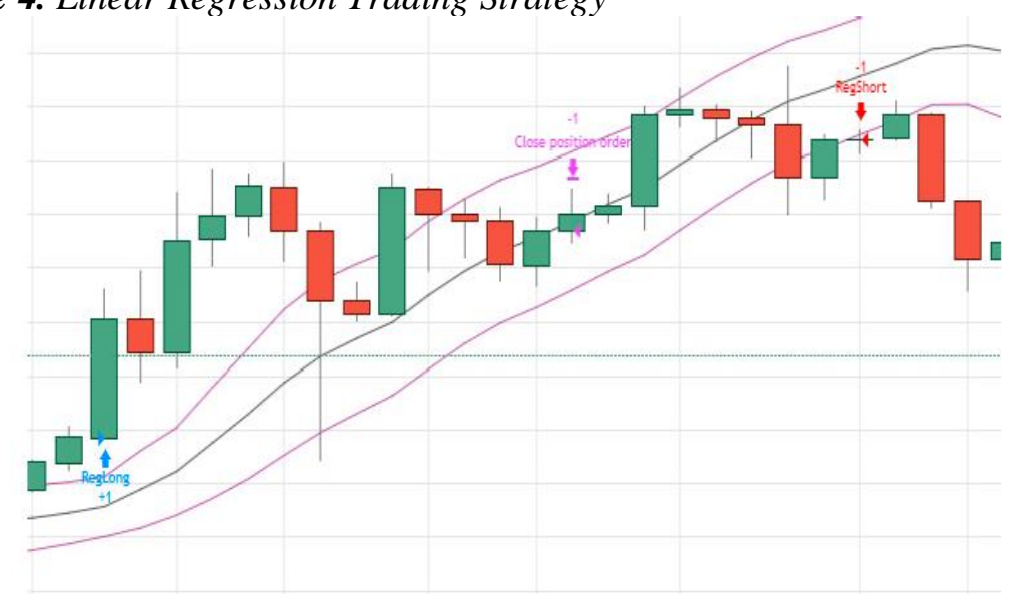

Source: Own study.

\subsection{Candlesticks Patterns}

Candlesticks are an important aspect of technical analysis. The advantage of candlesticks is that they produce trends shift signals with minimum lagging time in comparison to other technical tools. In the financial literature, the efficiency of candlesticks patterns remain debatable. Marshall et al. (2006; 2008) found that candlestick trading strategies do not have value for Dow Jones Industrial Average stocks or stocks that are traded on the Japanese stock market.

Heinz et al. (2020) performed a statistical analysis, using historical prices of the S\&P 500 index, of the effectiveness of bullish Engulfing and bearish Engulfing patterns. Their results indicate that the bearish Engulfing provide strong short-term forecasting power when using the open and high criteria but not the close criterion. Likewise, the bullish Engulfing offered strong short-term forecasting power when using the open and low criteria but not the close criterion. Horton (2009) observes nine candlestick patterns for 349 stocks listed in the S\&P 500 index and finds that the use of candlesticks patterns in trading individual stocks is not recommended.

Fock et al. (2005) examined the predictive power of candlesticks by employing intraday rather than daily data. They investigate 19 patterns and find negative results. Furthermore, they find that the forecasting power of candlesticks can be improved by combining oscillators that are based on technical analysis methods. Tsung et al. (2012) investigated two days reversal patterns in candlestick charting by using the Taiwan 50 component stocks. Out of six reversal patterns, they found that the bullish reversal patterns generally are more profitable than the bearish reversal patterns. Caginalp and Laurent (1998) argued that candlestick analysis has several advantages such as precise definitions of patterns and fixed time intervals of analysis. They found that most of the 3-day candlestick reversal patterns tested, 
appear to generate large profits. Nison (1991) and Pring (2002) stated that candlestick reversal patterns are notable when they occur in high-price or low-price areas.

Shiu and Lu (2011) employed daily data on Taiwan 69 electronic securities and found that the Bearish Harami pattern possesses genuine predictive power. Goo et al. (2007) compared average returns of various patterns and holding days and found that investors can gain an average return of $9.99 \%$ by using the bullish Harami pattern for a 10-day holding period. Meanwhile, the performance of candlesticks seems to be improved by implementing stop- loss strategies.

\subsubsection{Engulfing}

The Engulfing pattern is considered as one of the most powerful trend shift predictors. As can be seen in Figure 5, a bullish Engulfing pattern occur when a large green bar covers entirely a previous day red bar and a bearish Engulfing occurs when a red bar covers entirely a previous day red bar.

Figure 5. Engulfing Patterns Long and Short Signals

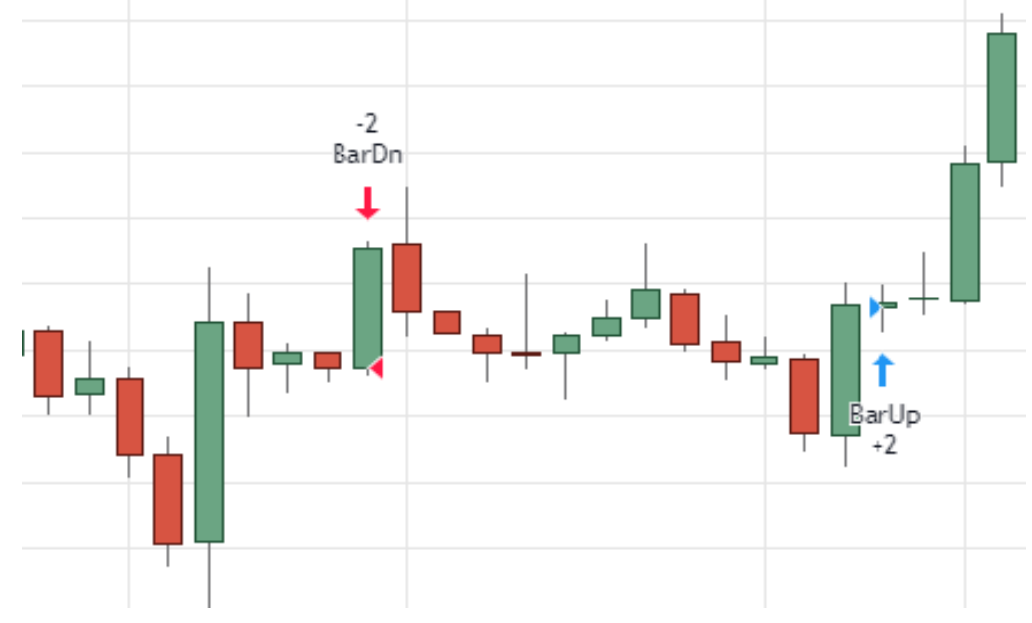

Source: Own study.

\subsubsection{Harami Patterns}

Like the Engulfing, an Harami pattern is also a two days pattern. A bullish Harami also occurs when a green bar follows a red bar. However, this time the red bar body covers entirely the following green bar. A bearish Harami occurs when a small red bar follows a large green bar that covers its body entirely (Figure 6). 
Figure 6. Harami Patterns Long and Short Signals

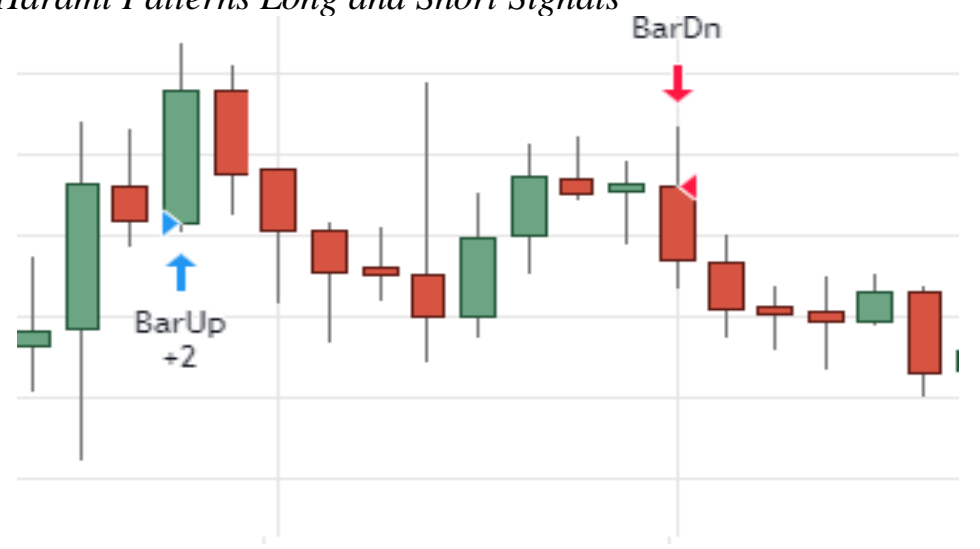

Source: Own study.

\section{Conclusions}

This paper discusses the major techniques that are used to construct algorithmic trading systems. We show that because of the complexity of the financial markets that reflects chaotic economics surrounding AL is needed for price forecasting of the different financial assets. The algorithmized "hands-free" trading systems have a huge advantage over human traders in their ability to integrate a large number of information sources into a single trading methodology.

The various sources included the past and current movement of the financial assets and external sources like social media and economic news suppliers. The ability of computerized algorithms to integrate a large set of data and react almost immediately does not come without risks of accelerating downtrends in times of panic in the financial market and therefore those systems must be institutionally monitored by the regulating authorities.

\section{References:}

Borovkova, S., Tsiamas, I. 2019. An ensemble of LSTM neural networks for high-frequency stock market classification. Journal of Forecasting. https://doi.org/10.1002/for.2585.

Caginalp, G., Laurent, H. 1998. The predictive power of price patterns. Applied Mathematical Finance, 5, 181-205.

Fischer, T., Krauss, C. 2018. Deep learning with long short-term memory networks for financial market prediction. European Journal of Operational Research, 270, 2, 654669.

Fock, J.H., Klein, C., Zwergel, B. 2005. Performance of candlestick analysis on intraday futures data. Journal of Futures Markets, 13, 28-40.

Goo, Y., Chen, D., Chang, Y. 2007. The application of Japanese candlestick trading strategies in Taiwan. Investment Management and Financial Innovations, 4, 49-71.

Heinz, A., Jamaloodeen, M., Sexena A., Pollacia, L. 2020. Bullish and Bearish Engulfing 
Japanese Candlestick patterns: A statistical analysis on the S\&P 500 index. The Quarterly Review of Economics and Finance.

Horton, M.J. 2009. Stars, crows, and doji: The use of candlesticks in stock selection. The Quarterly Review of Economics and Finance, 49, 283-294.

Liu, Y., Yang, A., Zhang, J., Jingjing, Y. 2020. An optimal stopping problem of detecting entry points for trading modeled by geometric Brownian motion. Computational Economics, 55, 827-843.

Marshall, B.R., Young, M.R., Rose, L.C. 2006. Candlestick technical trading strategies: Can they create value for investors? Journal of Banking and Finance, 30, 2303-2323.

Marshall, B.R., Young, M.R., Cahan, R. 2008. Are candlestick technical trading strategies profitable in the Japanese equity market? Review of Quantitative Finance and Accounting, 31, 191-207.

Nelson, D.M.Q., Pereira, A.C., de Oliveira, O. 2017. Stock market's price movement prediction with LSTM neural networks. International Joint Conference on Neural Networks (IJCNN), 1419-1426.

Nison, S. 1991. Japanese Candlestick Charting Techniques. New York, New York Institute of Finance.

Pring, M. 2002. Candlesticks Explained. New York, McGraw-Hill Trade.

Shiu, Y., Lu, T. 2011. Pinpoint and synergistic trading strategies of candlesticks. International Journal of Economics and Finance, 3, 234-244.

Tsung, H.L., Yung, M.S., Tsung, C.L. 2012. Profitable candlesticks trading strategies-the evidence from a new perspective. Review of Financial Economics, 21(2), 63-68.

Vapnik, V.N. 1995. The Nature of Statistical Learning Theory. Springer, New York, USA.

Xiao, C., Xia, W., Jiang, J. 2020. Stock price forecast based on combined model of ARIMA-LS-SVM. Neural Computing and Application, 32, 5379-5388. 